\title{
Rise and fall of outbreak-specific clone inside endemic pulsotype of Salmonella 4,[5],12:i:-; insights from high- resolution molecular surveillance in Emilia-Romagna, Italy, 2012 to 2015
}

\author{
Marina Morganti ${ }^{1}$, Luca Bolzoni ${ }^{2}$, Erika Scaltriti ${ }^{1}$, Gabriele Casadei ${ }^{1}$, Elena Carra ${ }^{3}$, Laura Rossi ${ }^{4}$, Paola Gherardi ${ }^{4}$, Fabio Faccini ${ }^{4}$, \\ Norma Arrigoni ${ }^{5}$, Anna Rita Sacchi ${ }^{4}$, Marco Delledonne ${ }^{4}$, Stefano Pongolini1, ${ }^{1,2}$ \\ 1. Istituto Zooprofilattico Sperimentale della Lombardia e dell’Emilia-Romagna, Sezione di Parma, Parma, Italy \\ 2. Istituto Zooprofilattico Sperimentale della Lombardia e dell'Emilia-Romagna, Risk Analysis Unit, Parma, Italy \\ 3. Istituto Zooprofilattico Sperimentale della Lombardia e dell'Emilia-Romagna, Sezione di Modena, Modena, Italy \\ 4. Local Health Unit of Piacenza, Department of Public Health, Piacenza, Italy \\ 5. Istituto Zooprofilattico Sperimentale della Lombardia e dell’Emilia-Romagna, Sezione di Piacenza, Gariga-Podenzano, Italy
}

Correspondence: Stefano Pongolini (stefano.pongolini@izsler.it)

Citation style for this article:

Morganti Marina, Bolzoni Luca, Scaltriti Erika, Casadei Gabriele, Carra Elena, Rossi Laura, Gherardi Paola, Faccini Fabio, Arrigoni Norma, Sacchi Anna Rita,

Delledonne Marco, Pongolini Stefano. Rise and fall of outbreak-specific clone inside endemic pulsotype of Salmonella 4,[5],12:i:-; insights from high-resolution

molecular surveillance in Emilia-Romagna, Italy, 2012 to 2015. Euro Surveill. 2018;23(13):pii=17-00375. https://doi.org/10.2807/1560-7917.ES.2018.23.13.17-00375

Background and aim: Epidemiology of human nontyphoid salmonellosis is characterised by recurrent emergence of new clones of the pathogen over time. Some clonal lines of Salmonella have shaped epidemiology of the disease at global level, as happened for serotype Enteritidis or, more recently, for Salmonella 4,[5],12:i:-, a monophasic variant of serotype Typhimurium. The same clonal behaviour is recognisable at sub-serotype level where single outbreaks or more generalised epidemics are attributable to defined clones. The aim of this study was to understand the dynamics of a clone of Salmonella 4, [5],12:i:over a 3-year period (2012-15) in a province of Northern Italy where the clone caused a large outbreak in 2013. Furthermore, the role of candidate outbreak sources was investigated and the accuracy of multilocus variable-number tandem repeat analysis (MLVA) was evaluated. Methods: we retrospectively investigated the outbreak through whole genome sequencing (WGS) and further monitored the outbreak clone for $\mathbf{2}$ years after its conclusion. Results: The study showed the transient nature of the clone in the population, possibly as a consequence of its occasional expansion in a food-processing facility. We demonstrated that important weaknesses characterise conventional typing methods applied to clonal pathogens such as Salmonella 4,[5],12:i:-, namely lack of accuracy for MLVA and inadequate resolution power for PFGE to be reliably used for clone tracking. Conclusions: The study provided evidence for the remarkable prevention potential of whole genome sequencing used as a routine tool in systems that integrate human, food and animal surveillance.

\section{Introduction}

Salmonellosis is among the most frequently reported food-borne diseases worldwide [1] and the second most-reported in the European Union (EU) [2]. In the past few decades, the epidemiology of salmonellosis has seen the emergence and/or expansion of specific types of Salmonella both at serotype and sub-serotype level [3]. In some instances, these newly-emerged types have gained wide and long-lasting geographic diffusion, as in the case of serotype Enteritidis, which gained worldwide epidemic diffusion in the 1980 s and 1990 [4]. More recently, Salmonella4,[5],12:i:- has been among the most frequently isolated serotypes from human cases in the EU [5-8]. The clonality of these emerging Salmonella has challenged the routinely-used typing methods and the effectiveness of laboratorybased surveillance $[9,10]$.

In this study, we retrospectively investigated a large outbreak by Salmonella 4,[5],12:i:- through whole genome sequencing (WGS) after it had become evident that conventional epidemiology and routine molecular methods, namely PFGE and multilocus variable-number tandem repeat analysis (MLVA), could not elucidate some critical aspects of the infectious episode. Furthermore, to accurately understand the temporal dynamics of the outbreak clone we extended WGS monitoring of the clone from 1 year before the outbreak onset to 2 years after its conclusion, i.e. from June 2012 to December 2015. The outbreak, which occurred in the Emilia-Romagna region of Italy in 2013, was detected by the routine regional surveillance system, based on PFGE. The epidemiological investigation 
Number of isolates per week of Salmonella 4,[5],12:i:- pulsotype STYMX.0131 in Piacenza province, Italy, 2012-2015 (n = 158)

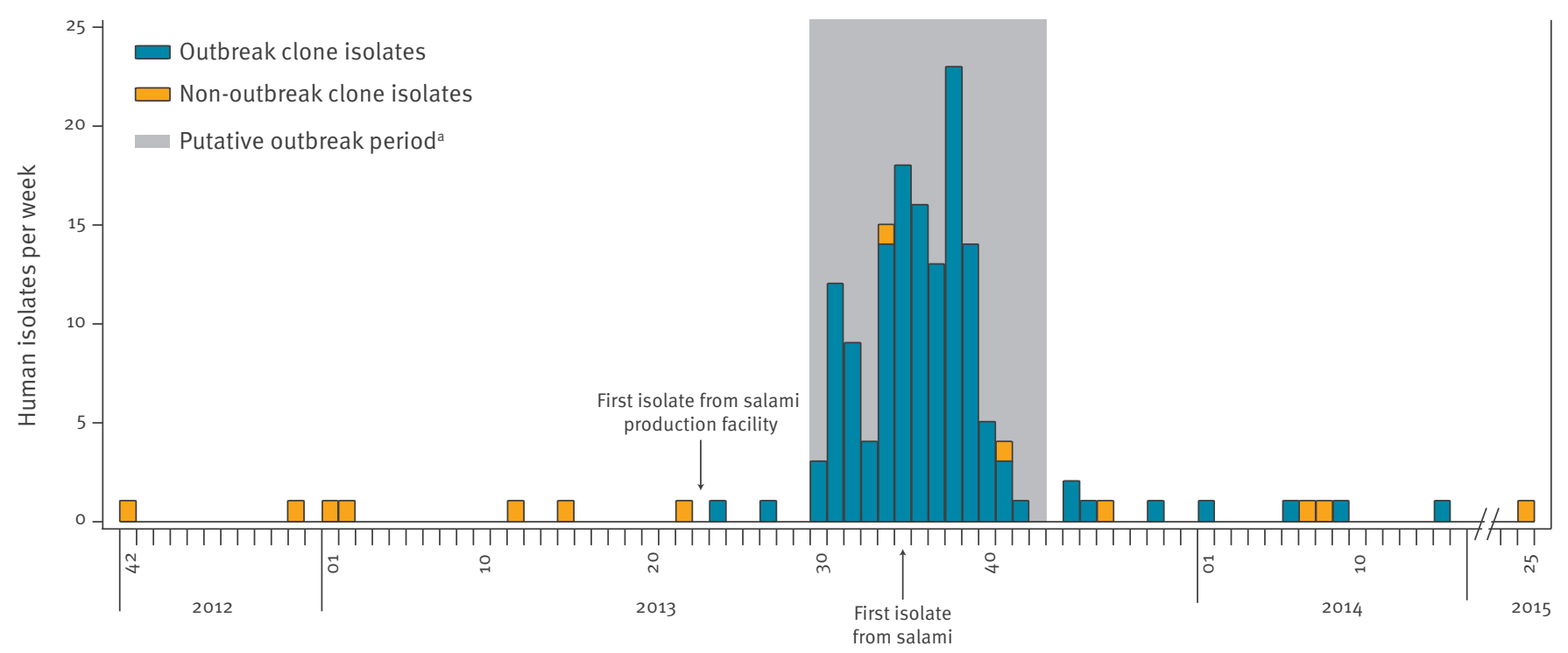

Week

${ }^{a}$ The putative outbreak period was defined based on the epidemiological evidence available.

rapidly identified the food involved (fermented drycured salami made from pork) and the facility implicated, but was not able to attribute several individuals infected with the implicated strain to the outbreak and could not confirm or exclude the role of suspect sources at abattoir and farm level. This was mainly the consequence of the outbreak pulsotype (STYMXB.0131) being the most common in the surveillance database and endemic in the area since at least $2008[8,11]$. More specifically, in the monitored pre-outbreak period (June 2012 to June 2013) the pulsotype represented $8.9 \%$ of all Salmonella isolates from humans and $19.2 \%$ of Salmonella 4,[5],12:i:-, in line with previous reports of this pulsotype in several European countries since the mid-2000s $[12,13]$. In particular, an extended Swiss study reported $37 \%$ prevalence of the pulsotype among human isolates of Samonella 4,[5],12:i:over the period 2007-11 [14]. Additional uncertainty during the outbreak investigation originated from the finding of variant types, although similar, among outbreak-related isolates, by PFGE (one variant) and, even more, by MLVA (five variants). The study was designed to address the unresolved issues and to answer specifically the following questions: (i) was there an outbreak-specific clone inside the endemic pulsotype STYMXB.0131 of Salmonella 4,[5],12:i:- and was it limited to the outbreak or circulating before and after it?; (ii) what was the exact responsibility of different candidate sources of the outbreak?; (iii) to what extent did the many observed MLVA variants belong to the outbreak clone? In other words, how accurate is MLVA in tracking Salmonella Typhimurium and variants in field conditions?; and (iv) what lessons can be learnt about the benefit of WGS as a surveillance tool in field conditions?

\section{Methods}

\section{Laboratory surveillance system for salmonellosis}

The laboratory surveillance system for human salmonellosis for Emilia-Romagna, an administrative region in Northern Italy with a population of ca 4.5 million residents [15], is a routine system based on serotyping and PFGE typing of all isolates recovered weekly from the regional network of medical microbiology laboratories. MLVA is performed on all isolates of $S$. Typhimurium and Salmonella 4,[5],12:i:- at a later stage (1-3 weeks later), as supplementary information to PFGE. To promptly identify potential outbreaks, a weekly analysis of the surveillance database is performed to assess whether any of the pulsotypes shows clustering in time beyond the expected threshold as previously described $[16,17]$. The outbreak alarm statistic is calculated with the OutbreakP algorithm from the 'surveillance' package in the R statistical environment [18]. The algorithm uses a weekly rolling window to evaluate whether the number of cases belonging to a given pulsotype is significantly greater than the expected number of cases during that period, based on the database of isolates starting from June 2012.

The system is integrated with the regional animal and foodstuff surveillance, which includes testing of 


\section{FIGURE 2}

Phylogenetic Bayesian tree of Salmonella 4,[5],12:i:- isolates sequenced in this study, Emilia-Romagna, Italy, 2012-2015

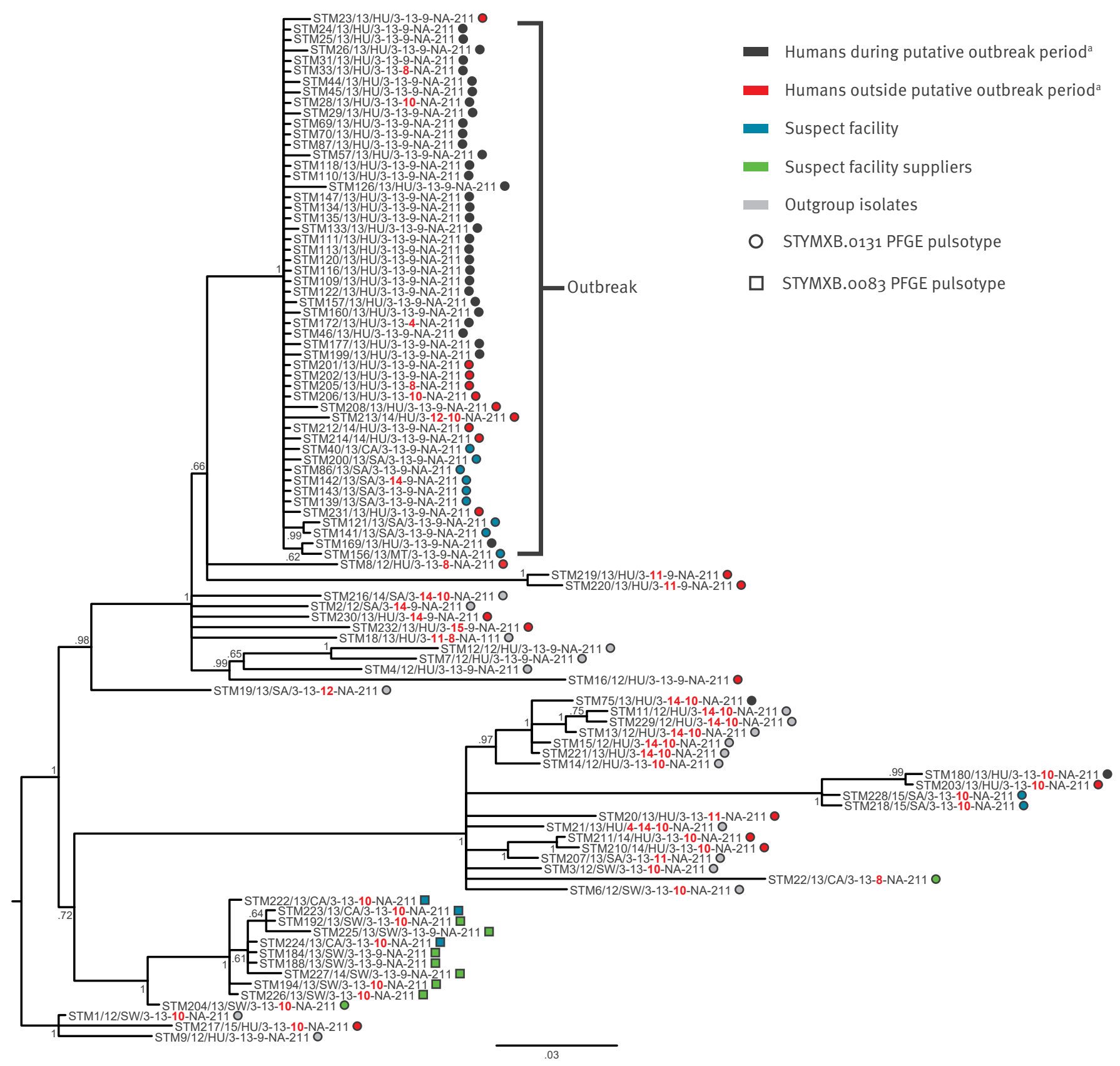

CA: carcass; HU: human; MLVA: multilocus variable-number tandem repeat analysis ; MT: meat; SA: salami; SW: swine.

a The putative outbreak period was defined based on the epidemiological evidence available.

The string identifying each isolate reports the study ID of the isolate, the year of isolation, the origin and MLVA profile ( 3 variant loci to the outbreak profile are in red).

official samples, private diagnostic activity on animals and some self-control testing by the food industry.

\section{Epidemiological investigation}

Case definition: following the alert for a possible outbreak of salmonellosis, a case was initially defined as any culture-confirmed human infection by Salmonella 4,[5],12:i:- with pulsotype STYMXB.0131, detected in the province of Piacenza after 22 July 2013. For the purpose of this study, the case definition included cases up to the epidemiologically defined end of the outbreak set at 29 October 2013. No cases other than laboratory-confirmed ones were considered within the scope of the study. 
Probability of occurrence of variant multilocus variable-number tandem repeat analysis profiles (panel A) and single nucleotide polymorphisms to the consensus (panel B) in outbreak isolates as a function of the isolation date

A.

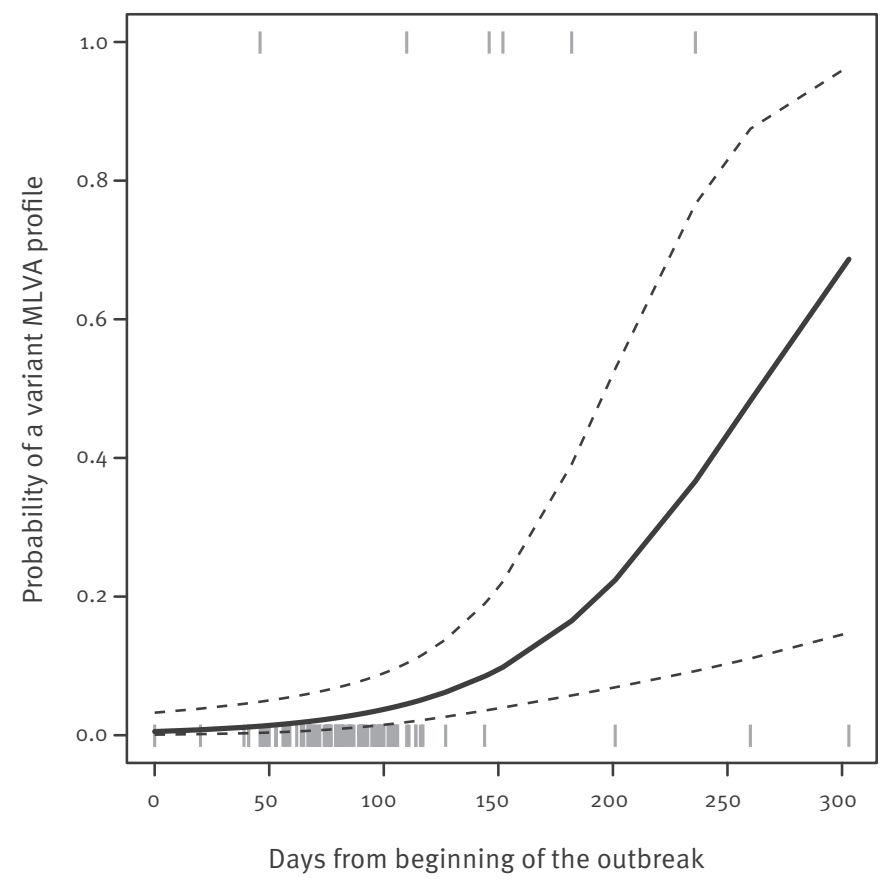

B.

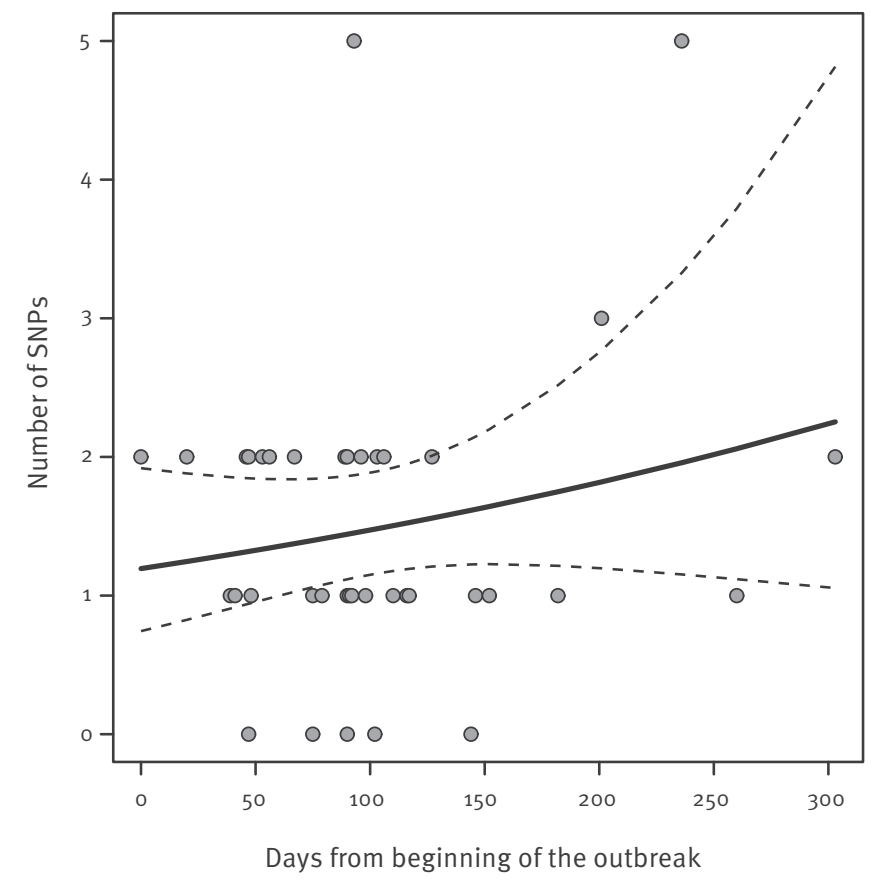

MVLA: multilocus variable-number tandem repeat analysis; SNPs: single nucleotide polymorphisms.

Generalised linear models with binomial distribution (panel A) or Poisson distribution (panel B). Solid black lines represent the best fit, dashed black lines represent $95 \%$ confidence intervals. In panel A, grey bars represent the occurrence (top bars) or not (bottom bars) of a variant MLVA profile in the isolate. In panel $B$, grey dots represent the number of SNPs to the consensus in the outbreak isolates.

Case interviews: the cases were interviewed by staff from the Local Health Unit of Piacenza using a standard format from the food-borne outbreaks reporting manual in accordance with directive 2003/99/EC from 2011 [19].

\section{Microbiological investigation}

Various types of samples were collected from the suspected sources during the outbreak investigation and tested for Salmonella. Sampling included salami, pork, the surface of pig carcasses, swine faeces, environmental swabs and faeces from the workers at the implicated salami-production facility. Foodstuffs were tested according to Regulation 2073/2005 [20]; for carcasses, four different sites of $100 \mathrm{~cm}^{2}$ each (hind limb medial, back, belly and cheek) were sampled using the abrasive sponge method. Swine faeces and boot swabs were tested with ISO 6579:2002/Amd.1: 2007 [21]. After the end of the outbreak, intensified monitoring of the salami-production facility was implemented within its hazard analysis and critical control points (HACCP) plan. The post-outbreak monitoring, from November 2013 to December 2015, included 123 batches of salami, 87 batches of raw meat, and 83 environmental swabs.
Typing of isolates and phylogenetic analysis After serotyping, the monophasic character of the isolates was confirmed by PCR as previously described [22]. PFGE was performed according to the PulseNet protocol with $\mathrm{Xbal}$ digestion of DNA [23] and restriction profiles were analysed by BioNumerics 7.5 (Applied Maths, Saint-Martens - Latem, Belgium). MLVA was performed as previously described [24] with a CEQ 8000 Genetic Analysis System (Beckman Coulter, US) and profiles were assigned according to the MLVA nomenclature suggested by Larsson et al. (2009) [25].

Ninety-eight isolates of the outbreak-related pulsotypes underwent WGS (Table 1), comprising a set of 19 diverse STYMXB.0131 isolates presumed to be unrelated to the outbreak and included in the analysis as outgroup isolates. To maximise the probability of these isolates not being outbreak-related, and therefore constituting adequate outgroup isolates, they were selected from human, animal and food isolates recovered from other parts of the region excluding Piacenza. They included a cluster of six isolates representing a small known outbreak that occurred in a nursery school 200 km from Piacenza in November 2012. 
Isolates of Salmonella 4,[5],12:i:-, study in Emilia-Romagna, Italy, 2012-2015 ( $\mathrm{n}=198$ )

\begin{tabular}{|c|c|c|c|c|c|c|}
\hline \multirow[b]{2}{*}{ Source } & \multirow{2}{*}{$\begin{array}{l}\text { Recovered } \\
\text { isolates }\end{array}$} & \multirow{2}{*}{$\begin{array}{l}\text { WGS-analysed } \\
\text { isolates }\end{array}$} & \multicolumn{2}{|c|}{ MLVA-variant isolates $^{a}$} & \multicolumn{2}{|c|}{ PFGE-variant isolates ${ }^{b}$} \\
\hline & & & $\begin{array}{l}\text { Number of } \\
\text { isolates }\end{array}$ & $\begin{array}{l}\text { Number of } \\
\text { types }\end{array}$ & $\begin{array}{l}\text { Number of } \\
\text { isolates }\end{array}$ & $\begin{array}{c}\text { Number of } \\
\text { types }\end{array}$ \\
\hline $\begin{array}{l}\text { Human (during putative outbreak } \\
\text { period) }\end{array}$ & 137 & $35^{c}$ & 5 & 4 & 0 & 0 \\
\hline $\begin{array}{l}\text { Human (outside putative outbreak } \\
\text { period) }\end{array}$ & 21 & 21 & 13 & 7 & 0 & 0 \\
\hline Salami production facility and salami & 14 & 14 & 6 & 2 & 3 & 1 \\
\hline Suppliers of salami production facility & 26 & $9^{d}$ & 5 & 2 & 7 & 1 \\
\hline Outgroup isolates & NA & 19 & 15 & 7 & 0 & 0 \\
\hline Total & 198 & 98 & 44 & NA & 10 & NA \\
\hline
\end{tabular}

MLVA: multilocus variable-number tandem repeat analysis; NA: not applicable; WGS: whole genome sequencing.

a With reference to outbreak type 3-13-9-NA-211; all variant isolates were WGS analysed.

${ }^{b}$ With reference to outbreak pulsotype STYMXB.0131; all variant isolates were WGS analysed.

'Isolates selected for WGS analysis were evenly distributed throughout the putative outbreak period.

d Including seven randomly selected STYMXB.0083 isolates of the 24 recovered from pig farm and the two STYMXB.0131 isolates recovered from pig farm and external slaughterhouse.

For WGS, sequencing libraries were prepared from genomic bacterial DNA with the Nextera XT sample preparation kit and run on an Illumina MiSeq (Illumina, San Diego, US) with a $2 \times 250$ paired-end run. The reads of the 98 genomes of Salmonella 4,[5],12:i:- were deposited at the European Bioinformatics Institute under Project Number PRJEB7560. The average sequencing coverage was $194 \mathrm{X}$ with $>75 \%$ of bases having Q value equal to or greater than 30. The genome of outbreak isolate STM45, used as reference for phylogenetic analysis, was assembled with MIRA 4.0 [26] using 'accurate settings for de novo assembly mode' and discarding small contigs (size $<1,000 \mathrm{bp}$ ). The average assembled-genome characteristics were: 5,010,485 nt length, 158X coverage, 144 contigs > 5,000 nt and N50 of 77,805 .

For phylogenetic analysis, the single nucleotide polymorphisms (SNPs) of each genome were extracted using a reference-based United States Food and Drug Administration SNP-pipeline (version 0.6.1), based on Bowtiez and VarScan [27]. The default settings of the pipeline were used for SNP calling, i.e. a minimum coverage of $8 x$ and a minimum variant allele frequency of 0.90. A Bayesian tree was generated with MRBAYES [28] from the SNP matrix of the analysed genomes. The Bayesian analysis was run using the GTR substitution model for 2,000,000 generations with chain sampled every 1,00 oth generation. The final parameter values and trees were summarised after discarding $25 \%$ of the posterior sample.

We assessed whether the occurrence of outbreak isolates with variant MLVA increased as a function of the isolation date by fitting a generalised linear model (GLM) with binomial error distribution. Similarly, we assessed whether the number of SNPs to the consensus, in the polymorphic positions shared by all outbreak-related human isolates, increased as a function of the isolation date by fitting a GLM with Poisson error distribution.

\section{Antimicrobial resistance and strain characterisation}

WGS-analysed isolates were tested for resistance to ampicillin, streptomycin, sulphonamides, tetracycline, gentamicin, chloramphenicol, enrofloxacin and cefotaxime by the disk diffusion susceptibility test [29]. The presence of antimicrobial resistance genes (ARG) was investigated in silico using the ResFinder database [30]. Mutations of gyrA-gyrB genes conferring resistance to quinolones were investigated by mapping the reads of the gyrA and $g y r B$ genes of all sequenced isolates on gyrA and gyrB nt sequences of sensitive $S$. Typhimurium LT2 [31]. The presence of Insertion Sequence 26 (IS26), known to drive genomic evolution linked to the monophasic character of 4,[5],12:i:strains [32], was investigated in the study genomes. To this end, sequencing reads were mapped on the biphasic $S$. Typhimurium $\mathrm{LT}_{2}$ reference genome (accession number AEo06468) and on the IS26 composite transposon regions of the previously characterised monophasic strains VAR-2009/08643/1 (accession number K999732) [32], 07-2006 (accession number KR856283) [11] and 105/7/03 (accession number HQ331538) [33]. To further explore the genomic differences between pulsotype STYMXB.0131 and its single-band variant STYMXB.0083, the SNPs exclusive to the STYMXB.0083 pulsotype were extracted and annotated using Prokka 1.7 [34]. Exclusive SNPs were defined as those present in all the genomes of a pulsotype and absent in all other genomes of the study. 
TABLE 2

Single nucleotide polymorphisms exclusive to the outbreak clone (detected in all the outbreak isolates and in no other isolate of the study), Emilia-Romagna, Italy, 2012-2015

\begin{tabular}{|c|c|c|c|c|c|c|c|c|}
\hline $\begin{array}{l}\text { Position in } \\
\text { reference }\end{array}$ & $\begin{array}{l}\text { Non- } \\
\text { outbreak Nt }\end{array}$ & Outbreak Nt & $\begin{array}{l}\text { Codon } \\
\text { change }\end{array}$ & $\begin{array}{l}\text { Aminoacid } \\
\text { change }\end{array}$ & Strand & Type of SNP & Gene & Product name \\
\hline 1005978 & A & G & $\begin{array}{l}\text { ATT } \cdots \cdot \dot{y} \\
\text { ATC }\end{array}$ & $1 \cdots+1$ & - & Synonymous & - & Short-chain dehydrogenase \\
\hline 1226252 & A & G & $\begin{array}{c}\text { TTG } \cdots \cdot \dot{*} \\
\text { CTG }\end{array}$ & $\mathrm{L} \cdots \cdot \mathrm{L}$ & - & Synonymous & mdtH & Multidrug resistance protein \\
\hline 2145307 & $C$ & $\mathrm{~T}$ & $\begin{array}{l}\mathrm{ACT} \cdots . . . \\
\text { ATT }\end{array}$ & $\mathrm{T} \cdots . . . \mathrm{Y} \mid$ & + & $\begin{array}{c}\text { Non- } \\
\text { synonymous }\end{array}$ & pduX & L-threonine kinase \\
\hline 2265999 & $\mathrm{~T}$ & C & $\begin{array}{c}\text { AAT } \cdots . . . \cdot \\
\text { GAT }\end{array}$ & $\mathrm{N} \cdots \cdots \cdot \mathrm{D}$ & - & $\begin{array}{c}\text { Non- } \\
\text { synonymous }\end{array}$ & yehU & Sensor histidine kinase \\
\hline 3526676 & A & $\mathrm{G}$ & $\begin{array}{l}\text { TAC } \ldots . . . \% \\
\text { TGC }\end{array}$ & $\mathrm{Y} \ldots . . . \mathrm{C}$ & + & $\begin{array}{c}\text { Non- } \\
\text { synonymous }\end{array}$ & acul & $\begin{array}{l}\text { Putative acrylyl-CoA } \\
\text { reductase }\end{array}$ \\
\hline 3912784 & $\mathrm{~T}$ & C & $\begin{array}{c}\mathrm{TGC} \ldots . . . \% \\
\mathrm{CGC}\end{array}$ & $\mathrm{C} \cdots \cdots \mathrm{G}$ & + & $\begin{array}{c}\text { Non- } \\
\text { synonymous }\end{array}$ & recG & $\begin{array}{c}\text { ATP-dependent DNA } \\
\text { helicase }\end{array}$ \\
\hline 4510357 & A & $\mathrm{C}$ & $\begin{array}{c}\mathrm{TGG} \ldots . . \\
\mathrm{GGG}\end{array}$ & $\mathrm{W} \ldots \mathrm{*} ; \mathrm{G}$ & - & $\begin{array}{c}\text { non- } \\
\text { synonymous }\end{array}$ & $\mathrm{fdhF}_{1}$ & Formate dehydrogenase \\
\hline
\end{tabular}

ATP: adenosine triphosphate; Nt: nucleotide; SNP: single nucleotide polymorphism.

\section{Results}

\section{Descriptive epidemiology}

The regional outbreak detection system signalled a potential outbreak by Salmonella 4,[5],12:i:- with pulsotype STYMXB.0131 on 14 August 2013. The first isolates with the outbreak pulsotype (OP) that were included in the alert by the signalling algorithm dated back to 22 July 2013. A total of 29 isolates with OP (each isolate corresponding to a different patient) had been recovered from the region between 22 July and 14 August. All but four of the isolates were from the province of Piacenza; therefore, it was considered that the potential outbreak concerned only that province, where the OP was increasing sharply while its incidence remained at the endemic level in the rest of the region where, overall, the OP was the most frequently reported pulsotype. The epidemic curve based on the case definition was steeply increasing, confirming the existence of the outbreak (Figure 1).

In particular, the average weekly number of isolates with the OP from Piacenza changed from 0.2 before the 22 July to 7.6 in the period 22 July to 14 August. The health authority was informed of the potential outbreak on 16 August. The interviews to the cases and their geographic restriction to a limited area of the province (in the north) rapidly led to the identification of a local facility producing fermented dry-cured salami made from pork as the possible source of the outbreak. The retail network of the producer overlapped with the location of the cases and the shop at the facility was mentioned repeatedly in case interviews. Microbiological confirmation of the facility as the source of the outbreak, interruption of distribution and recall of contaminated salami led to the reversion to the normal incidence of isolation of Salmonella 4,[5],12:i:- with OP and the outbreak was declared over on 29 October. Overall, 137 human isolates with OP were recovered from the outbreak territory between 22 July and 29 October. Based on the epidemiological evidence available, this time interval was identified as the putative outbreak period for the purpose of this study.

\section{Trace-back and environmental investigation} Inspection and microbiological investigation of the salami production facility demonstrated Salmonella 4,[5],12:i:- with OP in five of 21 tested batches of salami produced from 30 April to 10 September 2013 (representative isolates were: STM86; STM121; STM139; STM142; STM143; STM200). No batches processed before 30 April were still available for microbiology at the time of first inspection on 30 August. All environmental swabs $(n=50)$ were negative for Salmonella. The supply-chain information of the facility showed that some of the pork for salami production came from a small internal slaughterhouse at the facility and some was purchased from external industrial slaughterhouses. The pigs for the internal slaughterhouse originated predominantly from a single farm and to a lesser extent from other farms which supplied pigs occasionally.

Twenty-five faecal pools of 58 tested from the pen floors of the main pig-supplying farm were positive for Salmonella 4,[5],12:i:-, one had OP (STM204), all others had pulsotype STYMXB.0083, highly similar to the OP (representative isolates were: STM184; STM188; STM192; STM194; STM225; STM226). Pulsotype STYMXB.0083 was also detected on carcasses tested at the internal slaughterhouse and derived from pigs from that same farm (STM222; STM223; STM224). The facility's HACCP records reported that a carcass swab from the internal slaughterhouse had been 
found positive for Salmonella before outbreak onset, on 3 June 2013. Upon typing, the isolate showed a Salmonella 4,[5],12:i:- with OP (STM40). The carcass was from one of the farms which supplied pigs occasionally. The microbiological sampling of this farm (six environmental boot swabs) remained negative. As a result of the investigation and follow-up monitoring, Salmonella with OP was further detected. It was isolated from four of 22 healthy workers at the facility (STM133; STM134; STM135; STM172), from a batch of meat that had not yet entered salami processing in the internal slaughterhouse on 23 September (STM156) and from two batches of salami from the facility in January and March 2015 (STM218; STM228). Remarkably, one of the industrial slaughterhouses supplying meat to the facility had reported the presence of Salmonella 4,[5],12:i:- with OP (STM22) during its internal routine testing of carcass surfaces, before outbreak onset, on 28 June 2013.

\section{Molecular epidemiology}

All isolates included in the study had pulsotype STYMXB.0131 (part of the case definition) or its highly similar variant STYMXB.0083. As regards MLVA types, profile 3-13-9-NA-211 was detected in 132 isolates of 137 human isolates from the putative outbreak period, and was thus the most represented, but four other profiles were present among the remaining five isolates (STM28; STM33; STM75; STM172; STM180). Considering all human isolates eventually assigned to the outbreak cluster by WGS, four MLVA variants to 3-13-9-NA211 were detected, representing six isolates (STM28; STM33; STM172; STM205; STM206; STM213) (Figure 2).

The main outbreak profile (3-13-9-NA-211) was also detected among the isolates from salami, the internal slaughterhouse, the main pig-supplying farm and some of the outgroups of the phylogenetic analysis. Similarly to the human isolates, the isolates from the food chain showed a variety of MLVA profiles, generally closely related to profile 3-13-9-NA-211, consisting of singlelocus variants most of the time (Figure 2).

\section{Whole genome sequencing}

WGS analysis was performed to elucidate the individual responsibility of suspect sources along the food chain and to determine whether an outbreak-specific clone existed within the endemic STYMXB.0131 and what its time extension was. This high-resolution approach was required, given the existence of MLVA and PFGE variants among the outbreak isolates.

The outcome of the phylogenetic analysis is represented in Figure 2. Thirty-three of the 35 human isolates of the putative outbreak period, included in the analysis, belonged to the same clade that included the isolates from the four positive workers, all the isolates from salami collected during the investigation and the isolates from the internal slaughterhouse, STM40 (carcass before outbreak onset) and STM156 (meat for salami processing). No other isolates belonged to that clade except 10 humans out of the 21 recovered from Piacenza outside the putative outbreak period; the remaining 11 belonged to various different clades and were clearly not part of the outbreak. Seven SNPs were exclusive to and shared by all the outbreak isolates, supporting their cluster (Table 2).

Inside the outbreak clade, the number of SNPs to the consensus ranged from none to five (median value: one SNP), while the closest non-outbreak isolate (STM8) differed by 19 SNPs. As regards the responsibility of the food-chain operators, WGS confirmed the role of the salami producer, while both the main pig-supplying farm and the external slaughterhouse appeared to have no role in the contamination, different to what PFGE and MLVA testing had seemed to point to. In fact, all isolates with pulsotype STYMXB.0083 which came from the farm belonged to a well-segregated cluster in the phylogeny, distant from the outbreak clade (114 SNPS differed between the outbreak and the STYMXB.0083 consensus sequences), regardless of their PFGE and MLVA relatedness to the outbreak type. Similarly, the STYMXB.0131 isolates from the farm (STM204) and the external slaughterhouse (STM22) were shown to be distant from the outbreak clade. Remarkably, the isolates with OP from the salami facility of the follow-up monitoring were not related to the outbreak clone.

The phylogenetic analysis highlighted the clonality of the outgroup isolates from the 2012 nursery school outbreak, confirming the resolution power and accuracy of the analysis.

\section{Multilocus variable-number tandem repeat analysis variant analysis}

Statistical analyses revealed that the probability of an outbreak isolate being an MLVA variant increased at the end of the outbreak (GLM output: chi-squared $=8.04$, $D F=1, p=0.0046$ ), while the number of SNPs to the consensus did not significantly increase throughout the outbreak (GLM output: chi-squared $=1.65, D F=1$, $p=0.199$ ), see Figure 3 .

\section{Antimicrobial resistance and strain characterisation}

All sequenced isolates were tested for antimicrobial resistance and all but four were multidrug-resistant with R-type ASSuT (i.e. resistant to ampicillin, streptomycin, sulphonamides and tetracycline). The four exceptions did not belong to the outbreak clade and had ASSu R-type (STM2, STM3, STM232) or SuT R-type (STM8). The following ARGs were identified in all ASSUT isolates: blaTEM (ampicillin), strA and strB (streptomycin), sul2 (sulphonamides), tet(B) (tetracyclines). The three ASSu isolates lacked tet(B) and the SuT isolate lacked blaTEM and strB. No mutations associated with quinolone resistance were detected in gyrA and gyrB genes of the sequenced isolates. Similarly to other $4,[5], 12:$ : : strains $[11,32,33]$, both 
STYMXB.0131 and STYMXB.0083 isolates of the study possessed IS26 composite transposon insertions. Mapping of the sequencing reads on the $\mathrm{LT}_{2}$ reference genome revealed the deletion of STM2760-STM2772 chromosomal region in all sequenced genomes. This deletion, associated with IS26 insertions, corresponds to the loss of fljAB operon and hin gene (STM27702772 loci of $L T 2$ ), the genetic determinants of biphasic phenotype. The loss was consistent, in all study isolates, with the negative fljB-targeted PCR [35]. Further analysis of IS26 transposon insertions was done through comparison with the IS26 composite transposon regions of the previously characterised monophasic strains VAR-2009/08643/1 [32], 07-2006 [11] and 105/7/03 [33]. The comparison confirmed STM2760STM2772 deletion, as already observed in 07-2006, a strain with STYMXB.0131 pulsotype, where it was associated with the IS26-driven insertion of the RR3 element, a chromosomal module comprising various plasmid-derived genes and the ARGs blaTEM, strA, str $B$, sul2 and tet(B). Conversely, the study genomes did not have the STM2753-STM2759 deletion observed in 105/7/03, and they did not present the insertion of RR1 and RR2 elements around the fljAB operon described in VAR-2009/08643/1. Furthermore, all genes reported in the $\mathrm{RR}_{3}$ element of strain 07-2006 were detected in the study genomes with only five exceptions. These were represented by non-outbreak isolates lacking some of the RR3 genes, namely from tniA $\Delta$, to meth $\Delta$ genes (STM2, STM3, STM232); from tniA $\Delta$ to merR genes (STM20); from tnp2R $\Delta$ to $\operatorname{tnp} B$ and strB genes (STM8). Overall, these findings support the hypothesis that an RR3-like resistance element was present in STYMXB.0131 and STYMXB.0083 of the study in place of the STM2760-STM2772 region, similarly to strain 07-2006. This analysis showed that STYMXB.0131 and STYMXB.0083 from this study did not differ from one another as regards IS26-related genomic architecture and antibiotic resistance genes. Conversely, five synonymous SNPs located on a single gene, dus3, a putative tRNA-dihydrouridine synthase, were found to be exclusive to STYMXB.0083.

\section{Discussion}

\section{Antimicrobial resistance and strain}

\section{characterisation}

The ASSuT R-type and resistance genes of the outbreak $(\mathrm{OB})$ clone indicate its belonging to the clonal lineage of Salmonella 4,[5],12:i:- with pulsotype STYMXB.0131 and chromosomal multidrug resistance to ampicillin, streptomycin, sulphonamides and tetracycline commonly circulating in Europe [12]. The two related pulsotypes of the study showed the same genomic structure as regards the insertions of $\mathrm{IS}_{26} 6$, involved in their monophasic phenotype. That structure was already reported in Italian strains of pulsotype STYMXB.0131 collected between 2008 and 2012 [11], confirming the long-lasting circulation of this lineage in Italy. Nevertheless, according to WGS phylogeny, in our scenario, the minimum difference between the two PFGE profiles did correspond to great phylogenetic distance between the OB isolates (having STYMXB.0131 pulsotype) and the STYMXB.0083 isolates from the suspected farm. This distance is apparent from the tree topology of the study isolates and is substantiated by 114 SNPs of difference between the OB and the STYMXB.0083 consensus sequences.

\section{Outcome of the investigation}

WGS analysis accurately identified the existence of an outbreak-specific clone within the endemic pulsotype STYMXB.0131 of Salmonella 4,[5],12:i:- in the region affected. The 3-year long WGS monitoring extended the time boundaries of the outbreak beyond the originally identified (putative) outbreak period. Its duration was widened by 6 weeks before the epidemiologicallydefined onset as a consequence of the identification of two early $O B$ isolates and by 6 months after the apparent conclusion with the identification of eight late $\mathrm{OB}$ isolates (Figure 1). While the suspected salami was confirmed as the vehicle of infection, similarly to previous reports [36], the origin of the contamination upstream from the salami-processing facility was not demonstrated. The responsibility of the facility suppliers, initially suspected based on PFGE and MLVA, was not confirmed by WGS. In the absence of a demonstrated external origin of the contamination, this could be speculatively attributed to the positive healthy workers of the facility, although their positive status could be either the cause or the consequence of the salami contamination, with no possibility of solving the ambiguity. An alternative possible cause of the outbreak could have been persistent contamination inside the facility by the outbreak strain that could have entered the facility at some time before the outbreak, established itself and eventually contaminated the food products, reaching a level high enough to generate the observed high incidence of infections. The hypothesis of persistence is consistent with the sporadic isolation of the outbreak clone inside the facility before (STM40) and during the outbreak (STM156). Regardless of the origin of the contamination, the very limited number of SNPS differentiating the isolates of the $O B$ cluster and the lack of an evolutionary structure inside the cluster are indicative of a single, time-restricted source of the $O B$. This would be consistent with a sudden expansion of the contaminating clone inside the production premises, regardless of its origin (e.g. the facility environment, the raw meat or carrier workers). The in-depth cleaning of the facility, following the demonstration of its implication, probably led to the eradication of the strain from the processing plant, as confirmed by the negative results of the 2-year long post-outbreak monitoring on hundreds of samples. These results coincided with the rapid decline and the disappearance of the clone from the human population within 6 months of the official outbreak closure.

\section{Performance of typing methods}

Genomic investigation of the study outbreak through WGS confirmed, in a field scenario, that the high 
resolution of this approach allowed for the accurate assignment of the isolates to the outbreak clone, unlike current routine methods, PFGE and MLVA, which showed inadequate resolution and doubtful stability, respectively. This advantage of WGS, observed with a clonal pathogen like Salmonella 4,[5],12:i:- on a local geographical scale, could likely be helpful with clonal pathogens on larger geographical and temporal scales as well, where a greater number of variants can be expected with traditional typing methods. The use of WGS in widespread outbreaks, e.g. internationally, would imply sharing of standardised WGS data and analysis. In this study, MLVA showed a diversity of variants among the outbreak isolates that would potentially lead to incorrect assignment of some of the cases to the outbreak and to identify wrong links with suspected sources actually not related to the outbreak. This was the case for profiles 3-13-8-NA-211 and 3-13-10-NA-211 shared by some cases and suspected sources, which were eventually cleared of any responsibility. Even more misleading was the finding in the suspected pig farm of isolates having the main outbreak profile 3-13-9-NA-211 (STM184; STM188; STM225) which were eventually demonstrated not to belong to the outbreak clone. The results of other studies, conducted in recent years [8], and references thereof [37-39], are consistent with the ambiguity of MLVA evidenced by our data, despite the efforts of these authors to establish interpretation cut-offs for MLVA in terms of acceptable numbers of intra-outbreak repeats or variable loci. The high number of isolates analysed in our study and the complexity of the outbreak setting have shown limits of MLVA usage that those previous studies could not fully demonstrate. Interestingly, in our scenario the probability of emergence of MLVA variants was higher in the late phase of the outbreak, possibly reflecting mutation of the assayed loci with passing time at a rate higher than the rest of the genome, as measured through the accumulation of SNPs. While this is most likely not a limit in small and time-focused outbreaks, it could constitute a problem in lengthy ones.

\section{Lessons learnt}

The primary origin of the outbreak clone or its introduction into the region remained unexplained. However, the observed appearance and disappearance in the human population of the outbreak clone during a 3-year monitoring, its association with a specific food plant and the absence of identified primary sources, tell us that the emergence of a clone of Salmonella capable of generating a significant outbreak can indeed be a time-limited, transient event, possibly caused by extemporary clonal expansions inside food processing facilities, not necessarily with substantial quantitative contributions by upstream primary production.

From the methodological point of view, the study case showed that, based on WGS data, the first isolates unambiguously linking the salami facility (STM40) and the infection of humans (STM231) were available on 3 June and 13 June, respectively. This was more than a month in advance of the outbreak onset based on incidence (22 July) and more than 2 months in advance of the identification of the salami facility as the source of contamination (beginning of September). Retrospectively speaking, the availability of such genomic evidence in real-time or within the few days needed to produce it, would have favoured the adoption of mitigation measures with enough advance to prevent dozens of infections, indicating the preventive value of WGS if used routinely and promptly in surveillance systems combing human and food isolates. Although the identification of epidemiological links between clinical and food isolates will continue to be central in the attribution of outbreaks to their sources, the availability to the food control authorities of 'routine' food-human matching information can contribute to better planning and prioritisation of the control activity on facilities and food chains. These results contribute to a better understanding of the dynamics of Salmonella contamination at the food-human interface and highlighted the potential of WGS to improve the procedures of surveillance, investigation and trace-back of Salmonella as observed by others $[10,37,40-42]$.

\section{Acknowledgements}

The authors acknowledge the contribution of the Regional Health Service of Emilia-Romagna.

The study was partially funded by the Ministry of Health 'Ricerca Corrente' Grant N. IZSLER-PRC2013011 and N. IZSLER-PRC2014006.

\section{Conflict of interest}

None declared.

\section{Authors' contributions}

MM, LB, ES, GC and SP designed the study; MM performed the PFGE and laboratory surveillance; LB analysed the epidemiological data, did the statistical analyses and contributed to manuscript drafting; ES did the WGS, bioinformatic and phylogenetic analyses; GC contributed to bioinformatic and phylogenetic analyses, data collection and revision of results; EC performed MLVA and contributed to the revision of results; LR did the epidemiological data collection and contributed to the epidemiological investigation; PG performed data collection in support of microbiological investigation; FF performed epidemiological investigation and contributed to epidemiological data collection; NA performed the food and environmental microbiological investigations; ARS managed the public health surveillance system; MDD supervised the epidemiological investigation; SP coordinated the study and wrote the manuscript.

\section{References}

1. MajowiczSEMustoJ, ScallanE, AnguloFJ, KirkM, O’BrienSJ The global burden of nontyphoidal Salmonella gastroenteritis. Clin Infect Dis. 2010;50(6):882-9. DOI: 10.1086/650733 PMID: 20158401 
2. European Food Safety Authority (EFSA) / European Centre for Disease Prevention and Control (ECDC). The European Union summary report on trends and sources of zoonoses, zoonotic agents and food-borne outbreaks in 2013. EFSA J. 2015;13(1):3991. DOI: 10.2903/j.efsa.2015.3991

3. O'BrienSJ. The 'decline and fall' of nontyphoidal salmonella in the United Kingdom. Clin Infect Dis. 2013;56(5):705-10. DOI: 10.1093/cid/cis967 PMID: 23166188

4. WardLRThrelfall,, SmithHR, O'BrienSJ. Salmonella enteritidis epidemic. Science. 2000;287(5459):1753-4, author reply 17556. DOI: 10.1126/science.287.5459.1753C PMID: 10755925

5. European Food Safety Authority (EFSA) / European Centre for Disease Prevention and Control (ECDC). The European Union Summary Report on Trends and Sources of Zoonoses, Zoonotic Agents and Food-borne Outbreaks in 2012. EFSA J. 2014;12(2):3547. DOI: 10.2903/j.efsa.2014.3547

6. European Food Safety Authority (EFSA), European Centre for Disease Prevention and Control (ECDC). The European Union summary report on trends and sources of zoonoses, zoonotic agents and food-borne outbreaks in 2014. EFSA J. 2015;13(12):4329. DOI: 10.2903/j.efsa.2015.4329

7. CitoFBaldinelliF, CalistriP, Di GiannataleE, ScaviaG, OrsiniM Outbreak of unusual Salmonella enterica serovar Typhimurium monophasic variant 1,4 [5],12:i:-, Italy, June 2013 to September 2014. Euro Surveill. 2016;21(15):30194. DOI: 10.2807/15607917.ES.2016.21.15.30194 PMID: 27105170

8. BarcoLRamonE, CortiniE, LongoA, Dalla PozzaMC, LettiniAA Molecular characterization of Salmonella enterica serovar 4,[5],12:i:- DT193 ASSuT strains from two outbreaks in Italy. Foodborne Pathog Dis. 2014;11(2):138-44. DOI: 10.1089/ fpd.2013.1626 PMID: 24328499

9. OlsonABAndrysiakAK, TraczDM, Guard-BouldinJ, DemczukW, NgLK Limited genetic diversity in Salmonella enterica serovar Enteritidis PT13. BMC Microbiol. 2007;7(1):87. DOI: 10.1186/1471-2180-7-87 PMID: 17908316

10. AllardMWLuoY, StrainE, Pettengill], TimmeR, WangC On the evolutionary history, population genetics and diversity among isolates of Salmonella Enteritidis PFGE pattern JEGX01.0004. PLoS One. 2013;8(1):e55254. DOI: 10.1371/journal. pone.0055254 PMID: 23383127

11. GarcíaPMalornyB, RodicioMR, StephanR, HächlerH, GuerraB Horizontal Acquisition of a Multidrug-Resistance Module (R-type ASSUT) Is Responsible for the Monophasic Phenotype in a Widespread Clone of Salmonella Serovar 4,[5],12:i. Front Microbiol. 2016;7:680. DOI: 10.3389/fmicb.2016.00680 PMID: 27242707

12. HopkinsKLKirchnerM, GuerraB, GranierSA, LucarelliC, PorreroMC Multiresistant Salmonella enterica serovar 4,[5],12:i:- in Europe: a new pandemic strain? Euro Surveill. 2010;15(22):19580.PMID: 20546690

13. HauserETietzeE, HelmuthR, JunkerE, BlankK, PragerR Pork contaminated with Salmonella enterica serovar 4,[5],12:i:-, an emerging health risk for humans. Appl Environ Microbiol. 2010;76(14):4601-10. DOI: 10.1128/AEM.02991-09 PMID: 20472721

14. GallatiCStephanR, HächlerH, MalornyB, SchroeterA, NüeschInderbinenM. Characterization of Salmonella enterica subsp. enterica serovar 4,[5],12:i:- clones isolated from human and other sources in Switzerland between 2007 and 2011. Foodborne Pathog Dis. 2013;10(6):549-54. DOI: 10.1089/ fpd.2012.1407 PMID: 23614800

15. Italian National Institute of Statistics (Istat). Resident population on 1st January. Rome: Istat. [Accessed 27 Sep 2017]. Available from: http://dati.istat. it/Index.aspx?lang $=$ en $\&$ SubSessionld $=0579 \mathrm{eb}$ ed-fa5c-4c91-bc67-1ec73751e213\&themetreeid=21

16. FrisénMAnderssonE, PetterssonK. Semiparametric estimation of outbreak regression. Statistics. 2010;44(2):107-17. DOI: 10.1080/02331880903021484

17. TauxeRV. Molecular subtyping and the transformation of public health. Foodborne Pathog Dis. 2006;3(1):4-8. DOI: 10.1089/ fpd.2006.3.4 PMID: 16602974

18. Höhle M, Meyer S, Paul M. Surveillance: Temporal and SpatioTemporal Modelling and Monitoring of Epidemic Phenomena. 2015; R package version 1.9-1. [Accessed 9 Jun 2017]. Available from: http://CRAN.R-project.org/package=surveillance

19. European Food Safety Authority (EFSA). Manual for reporting on food-borne outbreaks in accordance with Directive 2003/99/EC for information derived from the year 2012. Parma: EFSA; 2012. Available from: http://onlinelibrary.wiley.com/ doi/10.2903/sp.efsa.2012.EN-265/epdf

20. European Commission. Regulation (EC) n. 2073/2005 on Microbiological criteria for foodstuffs. Luxembourg: Publications Office of the European Union. 22.12.2005:L 338/1. Available from: http://eur-lex.europa.eu/LexUriServ/ LexUriServ.do?uri=0J:L:2005:338:0001:0026:EN:PDF
21. International Organization for Standardization (ISO). Detection of Salmonella spp. in animal faeces and in environmental samples from the primary production stage. ISO 6579:2002/ Amd 1:2007. Geneva: ISO; July 2007. Available from: https:// www.iso.org/standard/42109.html

22. BarcollettiniAA, RamonE, LongoA, SaccardinC, PozzaMC $A$ rapid and sensitive method to identify and differentiate Salmonella enterica serotype Typhimurium and Salmonella enterica serotype 4,[5],12:i:- by combining traditional serotyping and multiplex polymerase chain reaction. Foodborne Pathog Dis. 2011;8(6):741-3. DOI: 10.1089/ fpd.2010.0776 PMID: 21247297

23. Centre for Disease Prevention and Control (CDC). Standard Operating Procedure for PulseNet PFGE of Escherichia coli 0157:H7, Escherichia coli non-0157 (STEC), Salmonella serotypes, Shigella sonnei and Shigella flexneri; Atlanta: CDC; 2013. Available from: http://www.cdc.gov/pulsenet/pdf/ecolishigella-salmonella-pfge-protocol-508c.pdf

24. LindstedtBAVardundT, AasL, KapperudG. Multiple-locus variable-number tandem-repeats analysis of Salmonella enterica subsp. enterica serovar Typhimurium using PCR multiplexing and multicolor capillary electrophoresis. J Microbiol Methods. 2004;59(2):163-72. DOI: 10.1016/j. mimet.2004.06.014 PMID: 15369852

25. LarssonJTTorpdahIM, PetersenRF, SorensenG, LindstedtBA, NielsenEM. Development of a new nomenclature for Salmonella typhimurium multilocus variable number of tandem repeats analysis (MLVA). Euro Surveill. 2009;14(15):14.PMID: 19371515

26. Chevreux B, Wetter T, Suhai S. Genome Sequence Assembly Using Trace Signals and Additional Sequence Information. Computer science and biology: Proceedings of German conference on bioinformatics (GCB); 1999.

27. PettengillJBLuoY, DavisS, ChenY, Gonzalez-EscalonaN, OttesenA An evaluation of alternative methods for constructing phylogenies from whole genome sequence data: a case study with Salmonella. Peer). 2014;2:e620. DOI: 10.7717/peerj.620 PMID: 25332847

28. HuelsenbeckJPRonquistF. MRBAYES: Bayesian inference of phylogenetic trees. Bioinformatics. 2001;17(8):754-5. DOI: 10.1093/bioinformatics/17.8.754 PMID: 11524383

29. BauerAWKirbyWM, SherrisJC, TurckM. Antibiotic susceptibility testing by a standardized single disk method. Am J Clin Pathol. 1966;45(4):493-6. DOI: 10.1093/ajcp/45.4_ts.493 PMID: 5325707

30. ZankariEHasmanH, CosentinoS, VestergaardM, RasmussenS, LundO Identification of acquired antimicrobial resistance genes. J Antimicrob Chemother. 2012;67(11):2640-4. DOI: 10.1093/jac/dks261 PMID: 22782487

31. HopkinsKLDaviesRH, ThrelfallEJ. Mechanisms of quinolone resistance in Escherichia coli and Salmonella: recent developments. Int J Antimicrob Agents. 2005;25(5):358-73. DOI: 10.1016/j.ijantimicag.2005.02.006 PMID: 15848289

32. BolandCBertrandS, MattheusW, DierickK, JassonV, RosseelT Extensive genetic variability linked to IS26 insertions in the fljB promoter region of atypical monophasic variants of Salmonella enterica serovar Typhimurium. Appl Environ Microbiol. 2015;81(9):3169-75. DOI: 10.1128/AEM.00270-15 PMID: 25724958

33. LucarelliCDionisiAM, FileticiE, OwczarekS, Luzzil, VillaL. Nucleotide sequence of the chromosomal region conferring multidrug resistance (R-type ASSuT) in Salmonella Typhimurium and monophasic Salmonella Typhimurium strains. J Antimicrob Chemother. 2012;67(1):111-4. DOI: 10.1093/jac/dkr391 PMID: 21990047

34. SeemannT. Prokka: rapid prokaryotic genome annotation. Bioinformatics. 2014;30(14):2068-9. DOI: 10.1093/ bioinformatics/btu153 PMID: 24642063

35. TennantSMDialloS, LevyH, LivioS, SowSO, TapiaM Identification by PCR of non-typhoidal Salmonella enterica serovars associated with invasive infections among febrile patients in Mali. PLoS Negl Trop Dis. 2010;4(3):e621. DOI: 10.1371/journal.pntd.0000621 PMID: 20231882

36. ScaltritiESasseraD, ComandatoreF, MorgantiM, MandalariC, GaiarsaS Differential single nucleotide polymorphism-based analysis of an outbreak caused by Salmonella enterica serovar Manhattan reveals epidemiological details missed by standard pulsed-field gel electrophoresis. J Clin Microbiol. 2015;53(4):1227-38. DOI: 10.1128/JCM.02930-14 PMID: 25653407

37. DimovskiKCaoH, WijburgOL, StrugnellRA, MantenaRK, WhippM Analysis of Salmonella enterica serovar Typhimurium variablenumber tandem-repeat data for public health investigation based on measured mutation rates and whole-genome sequence comparisons. J Bacteriol. 2014;196(16):3036-44. DOI: $10.1128 / J B .01820-14$ PMID: 24957617 
38. PetersenRFLitrupE, LarssonJT, TorpdahIM, SørensenG, MüllerL Molecular characterization of Salmonella Typhimurium

highly successful outbreak strains. Foodborne Pathog Dis.

2011;8(6):655-61. DOI: 10.1089/fpd.2010.0683 PMID: 21381921

39. WuytsVMattheusW, De Laminne de BexG, WildemauweC,

RoosensNH, MarchalK MLVA as a tool for public health

surveillance of human Salmonella Typhimurium: prospective study in Belgium and evaluation of MLVA loci stability. PLoS One. 2013;8(12):e84055. DOI: 10.1371/journal.pone.0084055 PMID: 24391880

40. LienauEKStrainE, WangC, ZhengJ, OttesenAR, KeysCE Identification of a salmonellosis outbreak by means of molecular sequencing. N Engl J Med. 2011;364(10):981-2. DOI: 10.1056/NEJMC1100443 PMID: 21345093

41. OctaviaSWangQ, TanakaMM, KaurS, SintchenkoV, LanR. Delineating community outbreaks of Salmonella enterica serovar Typhimurium by use of whole-genome sequencing: insights into genomic variability within an outbreak. J Clin Microbiol. 2015;53(4):1063-71. DOI: 10.1128/JCM.03235-14 PMID: 25609719

42. WuytsVDenayerS, RoosensNH, MattheusW, BertrandS,

MarchalK Whole Genome Sequence Analysis of Salmonella

Enteritidis PT4 Outbreaks from a National Reference

Laboratory's Viewpoint. PLoS Curr. 2015;7:10.PMID:

26468422

\section{License and copyright}

This is an open-access article distributed under the terms of the Creative Commons Attribution (CC BY 4.0) Licence. You may share and adapt the material, but must give appropriate credit to the source, provide a link to the licence, and indicate if changes were made.

This article is copyright of the authors, 2018. 